\title{
XCM Biologic Tissue Matrix xenograft and autologous micromucosa graft for vaginal reconstruction in Mayer-Rokitansky-Küster-Hauser syndrome
}

\author{
Tatiana Gigante Gomes ${ }^{1}$, Mariana Agostinho ${ }^{1}$, Mariana Conceição Cardoso ${ }^{2}$, \\ João Nunes da Costa $^{1}$, Júlio Matias ${ }^{1}$ \\ ${ }^{1}$ Department of Plastic, Reconstructive and Maxillofacial Surgery, Hospital of Egas Moniz, CHLO, Lisbon; ${ }^{2}$ Department of Obstetrics and \\ Gynecology, Hospital of Divino Espirito Santo of Ponta Delgada, EPER, Ponta Delgada, Portugal
}

\begin{abstract}
Mayer-Rokitansky-Küster-Hauser (MRKH) syndrome can be treated through numerous surgical and nonsurgical methods. We present a surgical technique in which a neovagina was reconstructed and shaped by a vaginal expander with acellular porcine dermal matrix (XCM Biologic Tissue Matrix) and mucosal interposition using microfragments harvested from the hymen. In our case, we found this procedure to be safe and effective, resulting in satisfactory sexual function and good cosmetic results, without donor site morbidity. To our best knowledge, this tissue-engineered biomaterial has never been used for vaginal reconstruction before.
\end{abstract}

Keywords Mayer-Rokitansky-Küster-Hauser syndrome / Vagina / Xenograft

\author{
Correspondence: \\ Tatiana Gigante Gomes \\ Department of Plastic, Reconstructive \\ and Maxillofacial Surgery, Hospital of \\ Egas Moniz, Rua Padre Américo no 4, \\ 30 Direito-Frente, 1600-548 Lisbon, \\ Portugal \\ Tel: +351-911971673 \\ Fax: +351-210432050 \\ E-mail: \\ Tatiana.gigante.gomes@gmail.com
}

Received: April 2, $2020 \bullet$ Revised: June 11, 2020 • Accepted: July 15, 2020

plSSN: 2234-6163・ elSSN: 2234-6171・https://doi.org/10.5999/aps.2020.00556• Arch Plast Surg 2021;48:185-188

\section{INTRODUCTION}

Mayer-Rokitansky-Küster-Hauser (MRKH) syndrome is a congenital malformation in which the Müllerian ducts fail to develop, resulting in aplasia/hypoplasia of the uterus and upper twothirds of the vagina in women with a 46,XX karyotype, normal secondary sexual characteristics, and normal ovarian function [1].

Treatment may be either nonsurgical, using vaginal expanders, or surgical, including full- or split-thickness skin grafts, local and locoregional flaps and bowel flaps [2]. The chosen method depends on many factors, such as the patient's motivation or preference and the surgeon's experience [3]. There is no consensus in the literature regarding the best option for surgical correction [4]. The Abbe-McIndoe technique, in which a split-thickness skin graft is used to cover the neovaginal canal lining, was a breakthrough in vaginoplasty [5]. Several modifications with many different materials have been proposed, including autologous buccal mucosa, artificial dermis, and in vitro vaginal cell cultures [6]. Taking into account the controversy about the ide- 
al material for vaginoplasty, here we present a surgical technique where a neovagina was reconstructed and shaped by a vaginal expander with acellular porcine dermal matrix (XCM Biologic Tissue Matrix) and mucosal interposition using microfragments harvested from the hymen. The anatomical and functional outcomes were satisfactory, and there were no visible scars at the donor site.

\section{CASE}

An 18-year-old woman with primary amenorrhea and inability of vaginal penetration presented with a seemingly normal vulva despite the nonexistence of the vaginal canal as assessed by a vaginal examination. Magnetic resonance imaging showed an atrophic uterus and vaginal aplasia, confirming the diagnosis of MRKH syndrome. Under general anesthesia, a U-shaped incision was made at the vulva just below the dimple (Fig. 1), three other incisions were made on the hymen at 3-, 6-, and 9-o'clock, and a potential space was created between the rectum and the bladder by careful blunt dissection while introducing a finger into the rectum to protect against injury. A cavity measuring $12-13 \mathrm{~cm}$ in length and about $4-5 \mathrm{~cm}$ in diameter was achieved. A neovagina was reconstructed with a vaginal expander (Silimed, Rio de Janeiro, Brazil) with acellular porcine dermal matrix (XCM Biologic Tissue Matrix; DePuy Synthes, Johnson \& Johnson, Solothurn, Switzerland) and mucosal interposition using microfragments harvested from the hymen. The xenograft, which measured $10 \times 13 \mathrm{~cm}$, was folded over itself lengthwise and a tube was created by suturing the edges together around the vaginal expander, which measured $12 \times 4 \mathrm{~cm}$, leaving an opening at one of the ends (Fig. 2). A piece of mucosa from the vaginal hymen was minced into pieces with diameters of 1-2

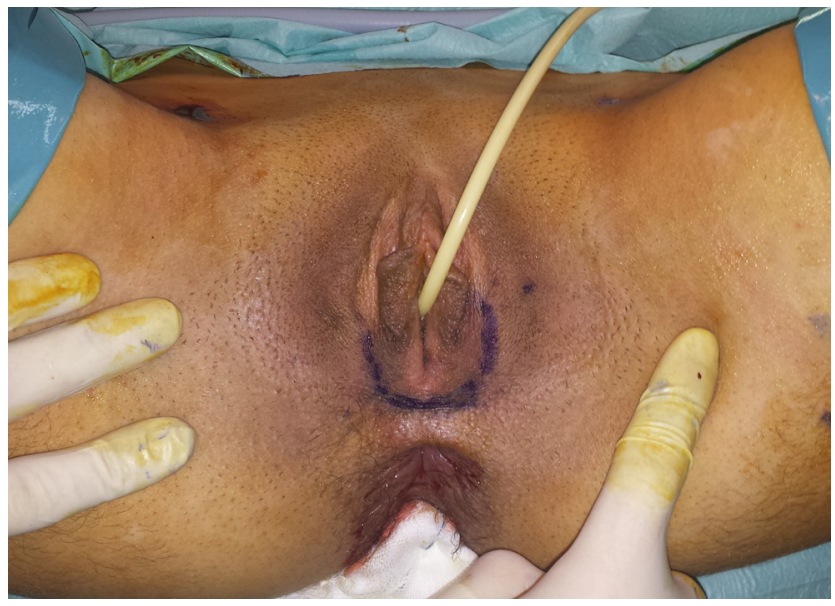

Fig. 1. Preoperative photograph. Preoperative marking a U-shaped incision in the vulva. $\mathrm{mm}$ and placed in the neovagina between the vaginal expander and the xenograft to promote epithelization (Fig. 3A-C). The xenograft and vaginal expander were inserted into the potential space, and the opening end of the XCM Biologic Tissue Matrix was sutured to the mucosa at the vaginal introitus with Vicryl 4/0 sutures. To prevent any hematoma or seroma, a JacksonPratt drain was also added (Fig. 3D). The patient was discharged from the hospital on the third postoperative day, after drain removal. The routine use of a vaginal expander to prevent stricture formation was performed and was progressively reduced until 6 months postoperatively, when the expander was totally discontinued and sexual intercourse was allowed to be initiated.

At a 4-year follow-up, the patient presented good aesthetic and functional results, reporting sexual intercourse without discomfort and frequent orgasms. The Female Sexual Function Index questionnaire showed good sexual satisfaction. Upon observation, the vagina and its mucosa were soft, pliable, and easily extended to $11 \mathrm{~cm}$ in length with a looseness of two finger-widths (Fig. 4).

\section{DISCUSSION}

When treating patients with MRKH syndrome, the primary goal is the creation of a functional neovagina that enables satisfactory sexual intercourse, thereby improving overall quality of life [7]. An ideal reconstruction should provide adequate dimensions, a physiological mucosal lining, and satisfying sexual function, along with minimal morbidity at the donor site [6]. Vaginoplasty using acellular dermal matrix presents many advantages when compared to the use of an autologous graft, such as the avoidance of scars and morbidity at the donor site, a
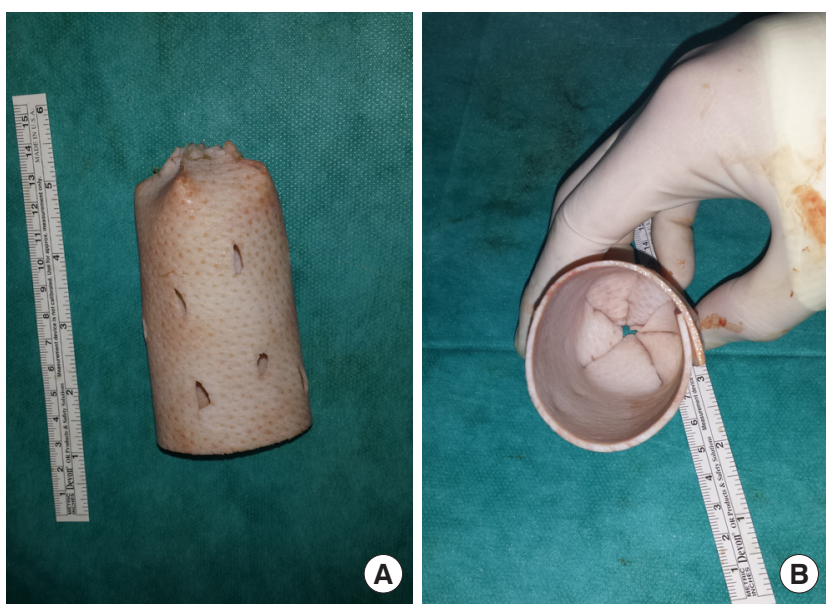

Fig. 2. Xenograft modeling. (A, B) XCM Biologic Tissue Matrix after being folded as a tube with an opening at one end. 

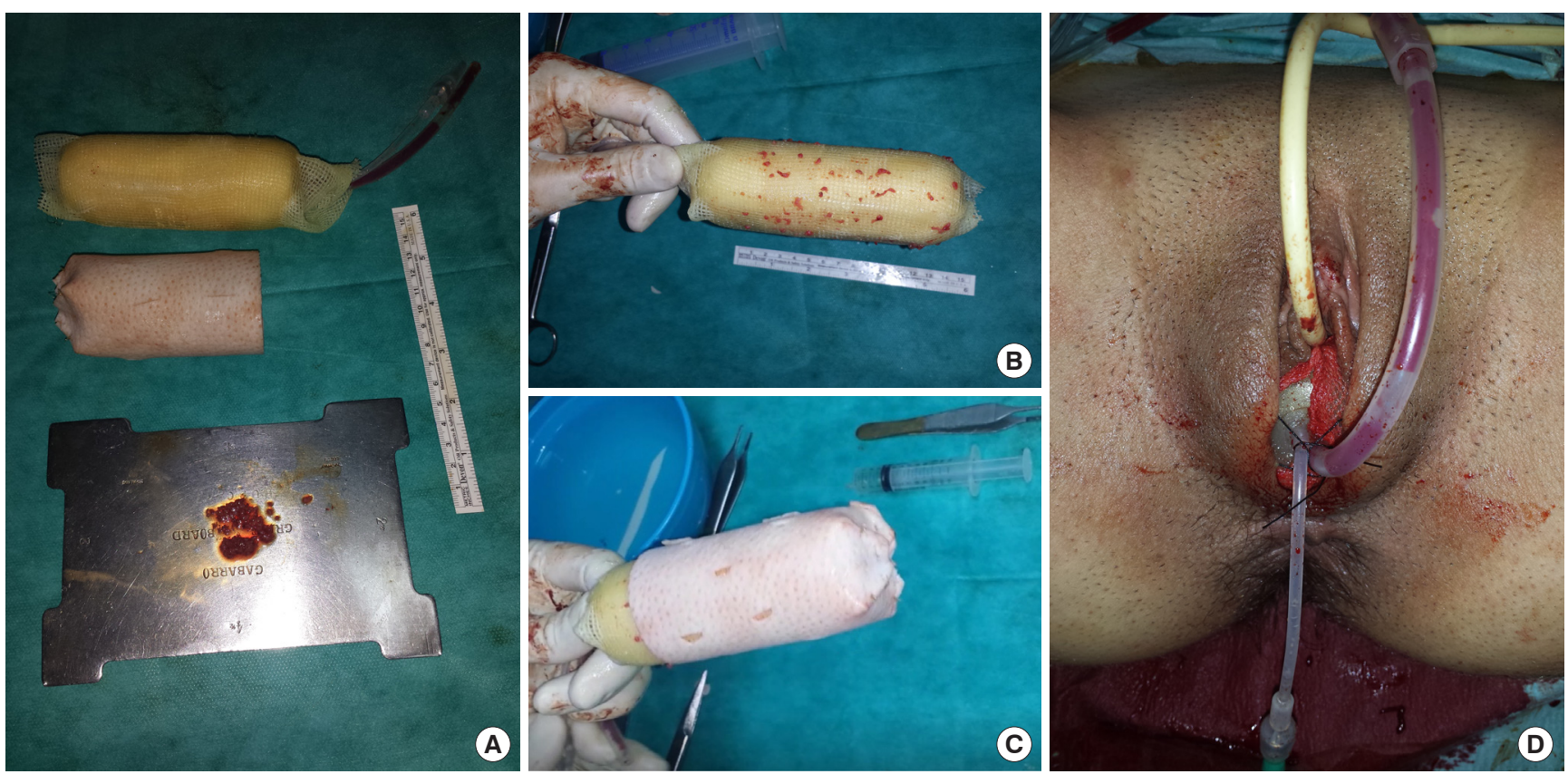

Fig. 3. Intraoperative photographs. (A) Vaginal expander mold, xenograft, and minced particles of mucosa harvested from the hymen. Mucosal particles adhered to the gauze around the vaginal mold (B), which were covered by the xenograft (C). (D) Immediate postoperative view.

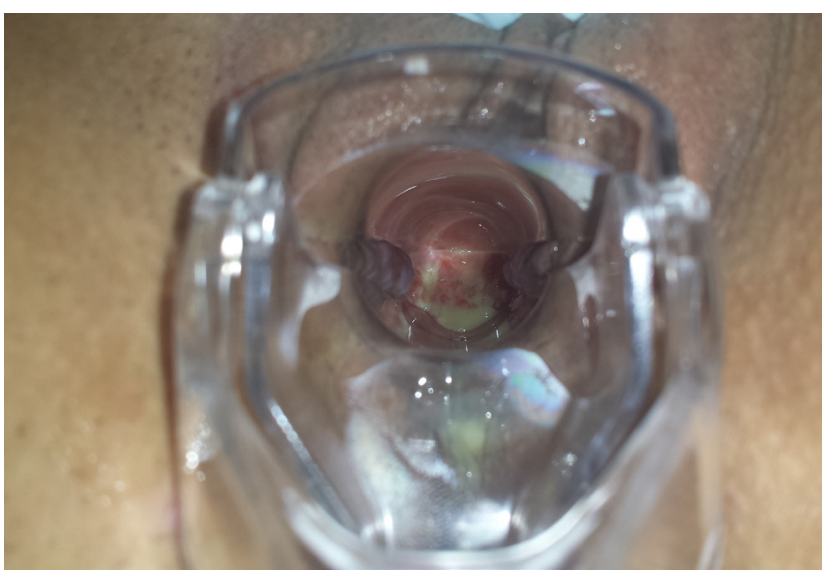

Fig. 4. Four years after the operation. The neovagina was well reconstructed with a soft, pliable, and easily extended mucosa lining.

shorter time for epithelization, the simplicity of the surgical technique, and also nearly normal sexual function outcomes [7].

The XCM Biologic Tissue Matrix is a sterile non-cross-linked three-dimensional matrix that is derived from porcine dermis. Following the Kensey Nash Optrix cleansing process, it becomes a strong and durable acellular biologic implant that serves as a scaffold for tissue regeneration, which is crucial for facilitating soft tissue healing, cell in-growth, and proliferation [8]. XCM Biologic Tissue Matrix has been mainly used in thoracic surgery [8-10], and there have been some in vivo studies in rabbits regarding its use in abdominal wall reconstruction, namely in ventral hernia repair [11]. Kaufmann et al. [12,13] performed experimental studies in rats regarding incisional hernia repair with biological meshes. Their study, using a peritonitis model, suggested that XCM Biologic Tissue Matrix was superior to other meshes in terms of incorporation, macroscopic mesh infection, and histological parameters including neovascularization and collagen deposition. The authors also advised overlapping the mesh to overcome the moderate shrinkage rate of XCM Biologic Tissue Matrix [12]. They also noted that this biomaterial had a much higher incorporation rate in a contaminated environment than in a physiological non-contaminated environment, suggesting this could be due to a higher fulminant inflammatory response [13].

Although no clinical cases have been published regarding the use of this biomaterial in vaginal reconstruction, we believe that XCM Biologic Tissue Matrix seems to be an adequate biological mesh to apply in vaginal surgery as it involves a contaminated field. In our case, the moderate shrinkage rate was overcome by using a vaginal expander for 6 months, and not by overlapping during placement.

Autologous micromucosa grafts were harvested from the hymen and introduced between the vaginal expander and the xenograft to promote epithelization. Taking into consideration the results presented by Teng et al. [6], we hypothesized that micromucosa interposition may have helped to physiologically reconstruct the new vagina with a mucosal lining, at the expense of an almost invisible scar and with tissue that would otherwise be 
disposable.

Although comparative evidence regarding various vaginoplasty methods has yet to emerge from randomized trials, we suggest that vaginoplasty using a xenograft is a safe, effective, and reliable technique that provides satisfactory sexual function and good cosmetic results without donor site morbidity [7].

To our best knowledge, this is the first case described of vaginal reconstruction with XCM Biologic Tissue Matrix, and we believe it deserves further attention because its maneuverability, plasticity, and tolerability make it favorable to apply in this anatomical area.

\section{NOTES}

\section{Conflict of interest}

No potential conflict of interest relevant to this article was reported.

\section{Ethical approval}

The study was approved by the CHLO Health Ethics Committee and performed in accordance with the principles of the Declaration of Helsinki. Written informed consent was obtained.

\section{Patient consent}

The patient provided written informed consent for the publication and the use of her images.

\section{Author contribution}

Conceptualization: T Gigante Gomes, M Agostinho, J Nunes da Costa, J Matias. Data curation, formal analysis, methodology, visualization: T Gigante Gomes. Writing - original draft: Gigante Gomes T, M Agostinho, MC Cardoso, J Nunes da Costa, J Matias. Writing - review \& editing: T Gigante Gomes, J Nunes da Costa, J Matias.

\section{ORCID}

Tatiana Gigante Gomes

https://orcid.org/0000-0002-5412-8670

Mariana Agostinho https://orcid.org/0000-0002-6471-3734 Mariana Conceição Cardoso https://orcid.org/0000-0002-9866-2584

João Nunes da Costa https://orcid.org/0000-0003-2412-4297 Júlio Matias https://orcid.org/0000-0003-3950-7573

\section{REFERENCES}

1. Oppelt PG, Lermann J, Strick R, et al. Malformations in a cohort of 284 women with Mayer-Rokitansky-Küster-Hauser syndrome (MRKH). Reprod Biol Endocrinol 2012;10:57.

2. Kim SK, Park JW, Lim KR, et al. Is rectosigmoid vaginoplasty still useful? Arch Plast Surg 2017;44:48-52.

3. Morcel K, Camborieux L; Programme de Recherches sur les Aplasies Müllériennes, et al. Mayer-Rokitansky-Küster-Hauser (MRKH) syndrome. Orphanet J Rare Dis 2007;2:13.

4. Committee on Adolescent Health Care. ACOG Committee Opinion No. 728: Müllerian Agenesis: Diagnosis, Management, And Treatment. Obstet Gynecol 2018;131:e35-42.

5. McIndoe A. The treatment of congenital absence and obliterative conditions of the vagina. Br J Plast Surg 1950;2:25467.

6. Teng Y, Zhu L, Chong Y, et al. The modified McIndoe technique: a scar-free surgical approach for vaginoplasty with an autologous micromucosa graft. Urology 2019;131:240-4.

7. Zhu L, Zhou H, Sun Z, et al. Anatomic and sexual outcomes after vaginoplasty using tissue-engineered biomaterial graft in patients with Mayer-Rokitansky-Küster-Hauser syndrome: a new minimally invasive and effective surgery. J Sex Med 2013;10:1652-8.

8. George RS, Kostopanagiotou K, Papagiannopoulos K. The expanded role of extracellular matrix patch in malignant and non-malignant chest wall reconstruction in thoracic surgery. Interact Cardiovasc Thorac Surg 2014;18:335-9.

9. Molinaro F, Garzi A, Cerchia E, et al. Sternal reconstruction by extracellular matrix: a rare case of phaces syndrome. Open Med (Wars) 2016;11:196-9.

10. Berthet JP, Wihlm JM, Canaud L, et al. The combination of polytetrafluoroethylene mesh and titanium rib implants: an innovative process for reconstructing large full thickness chest wall defects. Eur J Cardiothorac Surg 2012;42:444-53.

11. Pascual G, Sotomayor S, Adel F, et al. Remodeling of noncrosslinked acellular dermal matrices in a rabbit model of ventral hernia repair. Eur Surg Res 2016;56:32-48.

12. Kaufmann R, Jairam AP, Mulder IM, et al. Characteristics of different mesh types for abdominal wall repair in an experimental model of peritonitis. Br J Surg 2017;104:1884-93.

13. Kaufmann R, Jairam AP, Mulder IM, et al. Non-cross-linked collagen mesh performs best in a physiologic, noncontaminated rat model. Surg Innov 2019;26:302-11. 